\title{
Some new inequalities for the minimum eigenvalue of $M$-matrices
}

Feng Wang* and De-shu Sun

${ }^{\text {*Correspondence: }}$ wangf991@163.com

College of Science, Guizhou Minzu University, Guiyang, Guizhou 550025, P.R. China

\begin{abstract}
Some new inequalities for the minimum eigenvalue of $M$-matrices are obtained. These inequalities improve existing results, and the estimating formulas are easier to calculate since they only depend on the entries of matrices. Finally, some examples are also given to show that the bounds are better than some previous results.
\end{abstract}

MSC: $15 \mathrm{~A} 18 ; 15 \mathrm{~A} 42$

Keywords: M-matrix; nonnegative matrix; Hadamard product; spectral radius; minimum eigenvalue

\section{Introduction}

Let $\mathbb{C}^{n \times n}\left(\mathbb{R}^{n \times n}\right)$ denote the set of all $n \times n$ complex (real) matrices, $A=\left(a_{i j}\right) \in \mathbb{C}^{n \times n}, N=$ $\{1,2, \ldots, n\}$. We write $A \geq 0$ if all $a_{i j} \geq 0(i, j \in N)$. $A$ is called nonnegative if $A \geq 0$. Let $Z_{n}$ denote the class of all $n \times n$ real matrices all of whose off-diagonal entries are nonpositive. A matrix $A$ is called an $M$-matrix [1] if $A \in Z_{n}$ and the inverse of $A$, denoted by $A^{-1}$, is nonnegative. $M_{n}$ will be used to denote the set of all $n \times n M$-matrices.

Let $A$ be an $M$-matrix. Then there exists a positive eigenvalue of $A, \tau(A)=\rho\left(A^{-1}\right)^{-1}$, where $\rho\left(A^{-1}\right)$ is the spectral radius of the nonnegative matrix $A^{-1}, \tau(A)=\min \{|\lambda|: \lambda \in$ $\sigma(A)\}, \sigma(A)$ denotes the spectrum of $A . \tau(A)$ is called the minimum eigenvalue of $A[2,3]$. The Hadamard product of two matrices $A=\left(a_{i j}\right) \in \mathbb{R}^{n \times n}$ and $B=\left(b_{i j}\right) \in \mathbb{R}^{n \times n}$ is the ma$\operatorname{trix} A \circ B=\left(a_{i j} b_{i j}\right) \in \mathbb{R}^{n \times n}$.

An $n \times n$ matrix $A$ is said to be reducible if there exists a permutation matrix $P$ such that

$$
P^{T} A P=\left(\begin{array}{cc}
A_{11} & 0 \\
A_{21} & A_{22}
\end{array}\right),
$$

where $A_{11}, A_{22}$ are square matrices of order at least one. We call $A$ irreducible if it is not reducible. Note that any nonzero $1 \times 1$ matrix is irreducible.

Estimating the bounds for the minimum eigenvalue $\tau(A)$ of an $M$-matrix $A$ is an interesting subject in matrix theory, and it has important applications in many practical problems [4-6]. Hence, it is necessary to estimate the bounds for $\tau(A)$.

In [5], Shivakumar et al. obtained the following bound for $\tau(A)$ : Let $A=\left(a_{i j}\right) \in R^{n \times n}$ be a weakly chained diagonally dominant $M$-matrix. Then

$$
r(A) \leq \tau(A) \leq R(A), \quad \tau(A) \leq \min _{i \in N} a_{i i}, \quad \frac{1}{M} \leq \tau(A) \leq \frac{1}{m} .
$$

(c) 2015 Wang and Sun. This article is distributed under the terms of the Creative Commons Attribution 4.0 International License (http://creativecommons.org/licenses/by/4.0/), which permits unrestricted use, distribution, and reproduction in any medium, provided you give appropriate credit to the original author(s) and the source, provide a link to the Creative Commons license, and indicate if changes were made. 
Subsequently, Tian and Huang [7] provided a lower bound for $\tau(A)$ by using the spectral radius of the Jacobi iterative matrix $J_{A}$ of $A$ : Let $A=\left(a_{i j}\right) \in R^{n \times n}$ be an $M$-matrix and $A^{-1}=$ $\left(\alpha_{i j}\right)$. Then

$$
\tau(A) \geq \frac{1}{1+(n-1) \rho\left(J_{A}\right)} \frac{1}{\max _{i \in N}\left\{\alpha_{i i}\right\}} .
$$

Recently, Li et al. [8] improved (2) and gave the following result: Let $B=\left(b_{i j}\right) \in \mathbb{R}^{n \times n}$ be an $M$-matrix and $B^{-1}=\left(\beta_{i j}\right)$. Then

$$
\tau(B) \geq \frac{2}{\max _{i \neq j}\left\{\beta_{i i}+\beta_{i j}+\left[\left(\beta_{i i}-\beta_{j j}\right)^{2}+4(n-1)^{2} \beta_{i i} \beta_{j j} \rho^{2}\left(J_{B}\right)\right]^{\frac{1}{2}}\right\}} .
$$

In this paper, we continue to research the problems mentioned above. For an $M$-matrix $B$, we establish some new inequalities on the bounds for $\tau(B)$. Finally, some examples are given to illustrate our results.

For convenience, we employ the following notations throughout. Let $A=\left(a_{i j}\right)$ be an $n \times n$ matrix. For $i, j, k \in N, i \neq j$, denote

$$
\begin{aligned}
& r_{j i}=\frac{\left|a_{j i}\right|}{\left|a_{j j}\right|-\sum_{k \neq j, i}\left|a_{j k}\right|}, \quad r_{i}=\max _{j \neq i}\left\{r_{j i}\right\}, \\
& m_{j i}=\frac{\left|a_{j i}\right|+\sum_{k \neq j, i}\left|a_{j k}\right| r_{i}}{\left|a_{j j}\right|}, \quad h_{i}=\max _{j \neq i}\left\{\frac{\left|a_{j i}\right|}{\left|a_{j j}\right| m_{j i}-\sum_{k \neq j, i}\left|a_{j k}\right| m_{k i}}\right\}, \\
& u_{j i}=\frac{\left|a_{j i}\right|+\sum_{k \neq j, i}\left|a_{j k}\right| m_{k i} h_{i}}{\left|a_{j j}\right|}, \quad u_{i}=\max _{j \neq i}\left\{u_{i j}\right\} .
\end{aligned}
$$

\section{Main results}

In this section, we present our main results. Firstly, we give some notations and lemmas.

Let $A \geq 0$ and $D=\operatorname{diag}\left(a_{i i}\right)$. Denote $C=A-D, \mathcal{J}_{A}=D_{1}^{-1} C, D_{1}=\operatorname{diag}\left(d_{i i}\right)$, where

$$
d_{i i}= \begin{cases}1, & \text { if } a_{i i}=0 \\ a_{i i}, & \text { if } a_{i i} \neq 0\end{cases}
$$

By the definition of $\mathcal{J}_{A}$, we obtain

$$
\rho\left(\mathcal{J}_{A^{T}}\right)=\rho\left(D_{1}^{-1} C^{T}\right)=\rho\left(C D_{1}^{-1}\right)=\rho\left(D_{1}^{-1}\left(C D_{1}^{-1}\right) D_{1}\right)=\rho\left(D_{1}^{-1} C\right)=\rho\left(\mathcal{J}_{A}\right) .
$$

Lemma 1 [9] Let $A \in \mathbb{C}^{n \times n}$, and let $x_{1}, x_{2}, \ldots, x_{n}$ be positive real numbers. Then all the eigenvalues of $A$ lie in the region

$$
\bigcup_{i}\left\{z \in C:\left|z-a_{i i}\right| \leq x_{i} \sum_{j \neq i} \frac{1}{x_{j}}\left|a_{j i}\right|, i \in N\right\} .
$$

Lemma 2 [3] Let $A \in \mathbb{C}^{n \times n}$, and let $x_{1}, x_{2}, \ldots, x_{n}$ be positive real numbers. Then all the eigenvalues of $A$ lie in the region

$$
\bigcup_{j \neq i}\left\{z \in \mathbb{C}:\left|z-a_{i i}\right|\left|z-a_{j j}\right| \leq\left(x_{i} \sum_{k \neq i} \frac{1}{x_{k}}\left|a_{k i}\right|\right)\left(x_{j} \sum_{l \neq j} \frac{1}{x_{l}}\left|a_{l j}\right|\right)\right\} .
$$


Lemma 3 [3] Let $A, B \in \mathbb{R}^{n \times n}$, and let $X, Y \in \mathbb{R}^{n \times n}$ be diagonal matrices. Then

$$
X(A \circ B) Y=(X A Y) \circ B=(X A) \circ(B Y)=(A Y) \circ(X B)=A \circ(X B Y) .
$$

Lemma 4 [3] Let $A=\left(a_{i j}\right) \in M_{n}$. Then there exists a positive diagonal matrix $X$ such that $X^{-1} A X$ is a strictly diagonally dominant $M$-matrix

Lemma 5 [10] Let $A=\left(a_{i j}\right) \in R^{n \times n}$ be a strictly diagonally dominant matrix and let $A^{-1}=$ $\left(\alpha_{i j}\right)$. Then for all $i \in N$,

$$
\alpha_{i j} \leq u_{j i} \alpha_{j j}, \quad j \in N, j \neq i .
$$

Theorem 1 Let $A=\left(a_{i j}\right) \geq 0, B=\left(b_{i j}\right) \in M_{n}$, and let $B^{-1}=\left(\beta_{i j}\right)$. Then

$$
\rho\left(A \circ B^{-1}\right) \leq \max _{1 \leq i \leq n}\left\{\left(a_{i i}+u_{i} \rho\left(\mathcal{J}_{A}\right) d_{i i}\right) \beta_{i i}\right\}
$$

Proof It is evident that the result holds with equality for $n=1$.

We next assume that $n \geq 2$.

(i) First, we assume that $A$ and $B$ are irreducible matrices. Since $B$ is an $M$-matrix, by Lemma 4 , there exists a positive diagonal matrix $X$, such that $X^{-1} B X$ is a strictly row diagonally dominant $M$-matrix, and

$$
\rho\left(A \circ B^{-1}\right)=\rho\left(X^{-1}\left(A \circ B^{-1}\right) X\right)=\rho\left(A \circ\left(X^{-1} B X\right)^{-1}\right) .
$$

Hence, for convenience and without loss of generality, we assume that $B$ is a strictly diagonally dominant matrix.

On the other hand, since $\mathrm{A}$ is irreducible and so is $\mathcal{J}_{A^{T}}$. Then there exists a positive vector $x=\left(x_{i}\right)$ such that $\mathcal{J}_{A^{T}} x=\rho\left(\mathcal{J}_{A^{T}}\right) x=\rho\left(\mathcal{J}_{A}\right) x$, thus, we obtain $\sum_{j \neq i} a_{j i} x_{j}=\rho\left(\mathcal{J}_{A}\right) d_{i i} x_{i}$. Let $\widetilde{A}=\left(\tilde{a}_{i j}\right)=X A X^{-1}$ in which $X$ is the positive matrix $X=\operatorname{diag}\left(x_{1}, x_{2}, \ldots, x_{n}\right)$. Then, we have

$$
\widetilde{A}=\left(\tilde{a}_{i j}\right)=X A X^{-1}=\left(\begin{array}{cccc}
a_{11} & \frac{a_{12} x_{1}}{x_{2}} & \ldots & \frac{a_{1 n} x_{1}}{x_{n}} \\
\frac{a_{21} x_{2}}{x_{1}} & a_{22} & \ldots & \frac{a_{2 n} x_{2}}{x_{n}} \\
\vdots & \vdots & \ddots & \vdots \\
\frac{a_{n 1} x_{n}}{x_{1}} & \frac{a_{n 2} x_{n}}{x_{2}} & \ldots & a_{n n}
\end{array}\right)
$$

From Lemma 3, we have

$$
\widetilde{A} \circ B^{-1}=\left(X A X^{-1}\right) \circ B^{-1}=X\left(A \circ B^{-1}\right) X^{-1} .
$$

Thus, we obtain $\rho\left(\tilde{A} \circ B^{-1}\right)=\rho\left(A \circ B^{-1}\right)$. Let $\lambda=\rho\left(\widetilde{A} \circ B^{-1}\right)$, so that $\lambda \geq a_{i i} \beta_{i i}, \forall i \in N$. By Lemma 1 , there exists $i_{0} \in N$, such that

$$
\begin{aligned}
\left|\lambda-a_{i_{0} i_{0}} \beta_{i_{0} i_{0}}\right| & \leq u_{i_{0}} \sum_{t \neq i_{0}} \frac{1}{u_{t}} \tilde{a}_{t i_{0}} \beta_{t i_{0}} \leq u_{i_{0}} \sum_{t \neq i_{0}} \frac{1}{u_{t}} \tilde{a}_{t i_{0}} u_{t i_{0}} \beta_{i_{0} i_{0}} \\
& \leq u_{i_{0}} \sum_{t \neq i_{0}} \tilde{a}_{t i_{0}} \beta_{i_{0} i_{0}}=u_{i_{0}} \beta_{i_{0} i_{0}} \sum_{t \neq i_{0}} \frac{a_{t i_{0}} x_{t}}{x_{i_{0}}}=u_{i_{0}} \rho\left(\mathcal{J}_{A}\right) d_{i_{0} i_{0}} \beta_{i_{0} i_{0}} .
\end{aligned}
$$


Therefore,

$$
\lambda \leq a_{i_{0} i_{0}} \beta_{i_{0} i_{0}}+u_{i_{0}} \rho\left(\mathcal{J}_{A}\right) d_{i_{0} i_{0}} \beta_{i_{0} i_{0}}=\left(a_{i_{0} i_{0}}+u_{i_{0}} \rho\left(\mathcal{J}_{A}\right) d_{i_{0} i_{0}}\right) \beta_{i_{0} i_{0}},
$$

i.e.,

$$
\begin{aligned}
\rho\left(A \circ B^{-1}\right) & \leq\left(a_{i_{0} i_{0}}+u_{i_{0}} \rho\left(\mathcal{J}_{A}\right) d_{i_{0} i_{0}}\right) \beta_{i_{0} i_{0}} \\
& \leq \max _{1 \leq i \leq n}\left\{\left(a_{i i}+u_{i} \rho\left(\mathcal{J}_{A}\right) d_{i i}\right) \beta_{i i}\right\} .
\end{aligned}
$$

(ii) Now, assume that one of $A$ and $B$ is reducible. It is well known that a matrix in $Z_{n}$ is a nonsingular $M$-matrix if and only if all its leading principal minors are positive (see [1]). If we denote by $T=\left(t_{i j}\right)$ the $n \times n$ permutation matrix with $t_{12}=t_{23}=\cdots=t_{n-1, n}=t_{n 1}=-1$, the remaining $t_{i j}$ zero, then both $A-\varepsilon T$ and $B+\varepsilon T$ are irreducible matrices for any chosen positive real number $\varepsilon$, sufficiently small such that all the leading principal minors of $B+\varepsilon T$ are positive. Now, we substitute $A-\varepsilon T$ and $B+\varepsilon T$ for $A$ and $B$, respectively, in the previous case, and then letting $\varepsilon \rightarrow 0$, the result follows by continuity.

Theorem 2 Let $B=\left(b_{i j}\right) \in M_{n}$ and $B^{-1}=\left(\beta_{i j}\right)$. Then

$$
\tau(B) \geq \frac{1}{\max _{1 \leq i \leq n}\left\{\left(1+u_{i}(n-1)\right) \beta_{i i}\right\}} .
$$

Proof Let all entries of $A$ in (4) be 1 . Then $a_{i i}=1(\forall i \in N), \rho\left(\mathcal{J}_{A}\right)=n-1$. Therefore, by (4), we have

$$
\tau(B)=\frac{1}{\rho\left(B^{-1}\right)} \geq \frac{1}{\max _{1 \leq i \leq n}\left\{\left(1+u_{i}(n-1)\right) \beta_{i i}\right\}} .
$$

The proof is completed.

Theorem 3 Let $A=\left(a_{i j}\right) \geq 0, B=\left(b_{i j}\right) \in M_{n}$, and let $B^{-1}=\left(\beta_{i j}\right)$. Then

$$
\rho\left(A \circ B^{-1}\right) \leq \frac{1}{2} \max _{i \neq j}\left\{a_{i i} \beta_{i i}+a_{i j} \beta_{i j}+\Delta_{i j}\right\}
$$

where $\Delta_{i j}=\left[\left(a_{i i} \beta_{i i}-a_{j j} \beta_{j j}\right)^{2}+4 u_{i} u_{j} \rho^{2}\left(\mathcal{J}_{A}\right) d_{i i} d_{j j} \beta_{i i} \beta_{j j}\right]^{\frac{1}{2}}$.

Proof It is evident that the result holds with equality for $n=1$.

We next assume that $n \geq 2$. For convenience and without loss of generality, we assume that $B$ is a strictly row diagonally dominant matrix.

(i) First, we assume that $A$ and $B$ are irreducible matrices. Since $A$ is irreducible and so is $\mathcal{J}_{A^{T}}$. Then there exists a positive vector $y=\left(y_{i}\right)$ such that $\mathcal{J}_{A^{T}} y=\rho\left(\mathcal{J}_{A^{T}}\right) y=\rho\left(\mathcal{J}_{A}\right) y$, thus, we obtain

$$
\begin{aligned}
& \sum_{k \neq i} a_{k i} y_{k}=\rho\left(\mathcal{J}_{A}\right) d_{i i} y_{i}, \\
& \sum_{k \neq j} a_{k j} y_{k}=\rho\left(\mathcal{J}_{A}\right) d_{j j} y_{j} .
\end{aligned}
$$


Let $\widehat{A}=\left(\hat{a}_{i j}\right)=Y A Y^{-1}$ in which $Y$ is the positive matrix $Y=\operatorname{diag}\left(y_{1}, y_{2}, \ldots, y_{n}\right)$. Then, we have

$$
\widehat{A}=\left(\hat{a}_{i j}\right)=Y A Y^{-1}=\left(\begin{array}{cccc}
a_{11} & \frac{a_{12} y_{1}}{y_{2}} & \ldots & \frac{a_{1 n} y_{1}}{y_{n}} \\
\frac{a_{21} y_{2}}{y_{1}} & a_{22} & \ldots & \frac{a_{2 n} y_{2}}{y_{n}} \\
\vdots & \vdots & \ddots & \vdots \\
\frac{a_{n 1} y_{n}}{y_{1}} & \frac{a_{n 2} y_{n}}{y_{2}} & \ldots & a_{n n}
\end{array}\right) .
$$

From Lemma 3, we get

$$
\widehat{A} \circ B^{-1}=\left(Y A Y^{-1}\right) \circ B^{-1}=Y\left(A \circ B^{-1}\right) Y^{-1} .
$$

Thus, we obtain $\rho\left(\widehat{A} \circ B^{-1}\right)=\rho\left(A \circ B^{-1}\right)$. Let $\lambda=\rho\left(\widehat{A} \circ B^{-1}\right)$, so that $\lambda \geq a_{i i} \beta_{i i}(\forall i \in N)$. By Lemma 2 , there exist $i_{0}, j_{0} \in N, i_{0} \neq j_{0}$, such that

$$
\left|\lambda-a_{i_{0} i_{0}} \beta_{i_{0} i_{0}}\right|\left|\lambda-a_{j_{0 j}} \beta_{j_{0} j_{0}}\right| \leq\left(u_{i_{0}} \sum_{k \neq i_{0}} \frac{1}{u_{k}} \hat{a}_{k i_{0}} \beta_{k i_{0}}\right)\left(u_{j_{0}} \sum_{k \neq j_{0}} \frac{1}{u_{k}} \hat{a}_{k j_{0}} \beta_{k j_{0}}\right) .
$$

Note that

$$
\begin{aligned}
& u_{i_{0}} \sum_{k \neq i_{0}} \frac{1}{u_{k}} \hat{a}_{k i_{0}} \beta_{k i_{0}} \leq u_{i_{0}} \sum_{k \neq i_{0}} \frac{1}{u_{k}} \hat{a}_{k i_{0}} u_{k i_{0}} \beta_{i_{0} i_{0}} \leq u_{i_{0}} \beta_{i_{0} i_{0}} \sum_{k \neq i_{0}} \hat{a}_{k i_{0}}=u_{i_{0}} \beta_{i_{0} i_{0}} \rho\left(\mathcal{J}_{A}\right) d_{i_{0} i_{0}}, \\
& u_{j_{0}} \sum_{k \neq j_{0}} \frac{1}{u_{k}} \hat{a}_{k j_{0}} \beta_{k j_{0}} \leq u_{j_{0}} \sum_{k \neq j_{0}} \frac{1}{u_{k}} \hat{a}_{k j_{0}} u_{k j_{0}} \beta_{j_{0} j_{0}} \leq u_{j_{0}} \beta_{j_{0} j_{0}} \sum_{k \neq j_{0}} \hat{a}_{k j_{0}}=u_{j_{0}} \beta_{j_{0} j_{0}} \rho\left(\mathcal{J}_{A}\right) d_{j_{0} j_{0}} .
\end{aligned}
$$

Hence, we obtain

$$
\lambda \leq \frac{1}{2}\left(a_{i_{0} i_{0}} \beta_{i_{0} i_{0}}+a_{j_{0} j_{0}} \beta_{j_{0} j_{0}}+\Delta_{i_{0} j_{0}}\right)
$$

i.e.,

$$
\rho\left(A \circ B^{-1}\right) \leq \frac{1}{2}\left(a_{i_{0} i_{0}} \beta_{i_{0} i_{0}}+a_{j_{0} j_{0}} \beta_{j_{0} j_{0}}+\Delta_{i_{0} j_{0}}\right) \leq \frac{1}{2} \max _{i \neq j}\left\{a_{i i} \beta_{i i}+a_{j j} \beta_{j j}+\Delta_{i j}\right\}
$$

where $\Delta_{i j}=\left[\left(a_{i i} \beta_{i i}-a_{j j} \beta_{j j}\right)^{2}+4 u_{i} u_{j} \rho^{2}\left(\mathcal{J}_{A}\right) d_{i i} d_{j j} \beta_{i i} \beta_{j j}\right]^{\frac{1}{2}}$.

(ii) Now, assume that one of $A$ and $B$ is reducible. We substitute $A-\varepsilon T$ and $B+\varepsilon T$ for $A$ and $B$, respectively, in the previous case (as in the proof of Theorem 1), and then letting $\varepsilon \rightarrow 0$, the result follows by continuity.

Theorem 4 Let $B=\left(b_{i j}\right) \in M_{n}$ and $B^{-1}=\left(\beta_{i j}\right)$. Then

$$
\tau(B) \geq \frac{2}{\max _{i \neq j}\left\{\beta_{i i}+\beta_{j j}+\Delta_{i j}\right\}},
$$

where $\Delta_{i j}=\left[\left(\beta_{i i}-\beta_{i j}\right)^{2}+4(n-1)^{2} u_{i} u_{j} \beta_{i i} \beta_{j j}\right]^{\frac{1}{2}}$. 
Proof Let all entries of $A$ in (6) be 1. Then

$$
a_{i i}=1 \quad(\forall i \in N), \quad \rho\left(\mathcal{J}_{A}\right)=n-1, \quad \Delta_{i j}=\left[\left(\beta_{i i}-\beta_{i j}\right)^{2}+4(n-1)^{2} u_{i} u_{j} \beta_{i i} \beta_{j j}\right]^{\frac{1}{2}} .
$$

Therefore, by (6), we have

$$
\tau(B)=\frac{1}{\rho\left(B^{-1}\right)} \geq \frac{2}{\max _{i \neq j}\left\{\beta_{i i}+\beta_{j j}+\Delta_{i j}\right\}} .
$$

The proof is completed.

Remark 1 We next give a simple comparison between (4) and (6), (5) and (7), respectively. For convenience and without loss of generality, we assume that, for $i, j \in N, i \neq j$,

$$
a_{j j} \beta_{j j}+u_{j} d_{j j} \beta_{j j} \rho\left(\mathcal{J}_{A}\right) \leq a_{i i} \beta_{i i}+u_{i} d_{i i} \beta_{i i} \rho\left(\mathcal{J}_{A}\right)
$$

i.e.,

$$
u_{j} d_{j j} \beta_{i j} \rho\left(\mathcal{J}_{A}\right) \leq a_{i i} \beta_{i i}-a_{j j} \beta_{j j}+u_{i} d_{i i} \beta_{i i} \rho\left(\mathcal{J}_{A}\right) .
$$

Hence,

$$
\begin{aligned}
\Delta_{i j} & =\left[\left(a_{i i} \beta_{i i}-a_{j j} \beta_{j j}\right)^{2}+4 u_{i} u_{j} \rho^{2}\left(\mathcal{J}_{A}\right) d_{i i} d_{j j} \beta_{i i} \beta_{i j}\right]^{\frac{1}{2}} \\
& \leq\left[\left(a_{i i} \beta_{i i}-a_{j j} \beta_{j j}\right)^{2}+4 u_{i} \rho\left(\mathcal{J}_{A}\right) d_{i i} \beta_{i i}\left(a_{i i} \beta_{i i}-a_{j j} \beta_{j j}+u_{i} d_{i i} \beta_{i i} \rho\left(\mathcal{J}_{A}\right)\right)\right]^{\frac{1}{2}} \\
& =a_{i i} \beta_{i i}-a_{j j} \beta_{i j}+2 u_{i} d_{i i} \beta_{i i} \rho\left(\mathcal{J}_{A}\right) .
\end{aligned}
$$

Further, we obtain

$$
a_{i i} \beta_{i i}+a_{j j} \beta_{j j}+\Delta_{i j} \leq 2 a_{i i} \beta_{i i}+2 u_{i} d_{i i} \beta_{i i} \rho\left(\mathcal{J}_{A}\right),
$$

i.e.,

$$
\rho\left(A \circ B^{-1}\right) \leq \frac{1}{2} \max _{i \neq j}\left\{a_{i i} \beta_{i i}+a_{j j} \beta_{j j}+\Delta_{i j}\right\} \leq \max _{1 \leq i \leq n}\left\{\left(a_{i i}+u_{i} \rho\left(\mathcal{J}_{A}\right) d_{i i}\right) \beta_{i i}\right\} .
$$

So, the bound in (6) is better than the bound in (4). Similarly, we can prove that the bound in (7) is better than the bound in (5).

\section{Numerical examples}

In this section, we present numerical examples to illustrate the advantages of our derived results.

Example 1 Let

$$
B=\left(\begin{array}{ccc}
1.1 & -0.6 & -0.1 \\
-0.3 & 1 & -0.6 \\
-0.2 & -0.4 & 0.7
\end{array}\right) .
$$


It is easy to see that $B$ is an $M$-matrix. By calculations with Matlab 7.1, we have

$$
\begin{aligned}
& \tau(B) \geq 0.10000000 \quad(\text { by }(1)), \quad \tau(B) \geq 0.11396723 \quad \text { (by }(2)) \text {, } \\
& \tau(B) \geq 0.11582163 \quad(\text { by }(3)), \quad \tau(B) \geq 0.11834016 \quad \text { (by (5)), } \\
& \tau(B) \geq 0.13163534 \quad \text { (by }(7)) \text {, }
\end{aligned}
$$

respectively. In fact, $\tau(B)=0.16213193$. It is obvious that the bound in (7) is the best result.

\section{Example 2 Let}

$$
B=\left(\begin{array}{ccccc}
1 & -0.2 & -0.1 & -0.2 & -0.1 \\
-0.4 & 1 & -0.2 & -0.1 & -0.1 \\
-0.3 & -0.2 & 1 & -0.1 & -0.1 \\
-0.2 & -0.3 & -0.3 & 1 & -0.1 \\
-0.1 & -0.3 & -0.2 & -0.2 & 1
\end{array}\right) \text {. }
$$

It is easy to see that $B$ is an $M$-matrix. By calculations with Matlab 7.1, we have

$$
\begin{aligned}
& \tau(B) \geq 0.10000000 \quad(\text { by }(1)), \quad \tau(B) \geq 0.16082517 \quad(\text { by }(2)), \\
& \tau(B) \geq 0.16831778 \quad(\text { by }(3)), \quad \tau(B) \geq 0.18147932 \quad \text { (by (5)), } \\
& \tau(B) \geq 0.19169108 \quad(\text { by }(7)) \text {, }
\end{aligned}
$$

respectively. In fact, $\tau(B)=0.25807710$. It is obvious that the bound in (7) is the best result.

\section{Competing interests}

The authors declare that they have no competing interests.

\section{Authors' contributions}

The authors contributed equally to this work. All authors read and approved the final manuscript.

\section{Acknowledgements}

The authors are very indebted to the referees for their valuable comments and corrections, which improved the original manuscript of this paper. This work was supported by National Natural Science Foundations of China (11361074, 71161020), Applied Basic Research Programs of Science and Technology Department of Yunnan Province (2013FD002), and IRTSTYN, Research Foundation of Guizhou Minzu University (15XRY004).

Received: 21 April 2015 Accepted: 1 June 2015 Published online: 16 June 2015

\section{References}

1. Berman, A, Plemmons, RJ: Nonnegative Matrices in the Mathematical Sciences. Academic Press, New York (1979)

2. Fiedler, M, Markham, T: An inequality for the Hadamard product of an M-matrix and inverse M-matrix. Linear Algebra Appl. 101, 1-8 (1988)

3. Horn, RA, Johnson, CR: Topics in Matrix Analysis. Cambridge University Press, Cambridge (1991)

4. Corduneanu, C: Principles of Differential and Integral Equations. Chelsea, New York (1988)

5. Shivakumar, PN, Williams, JJ, Ye, Q, Marinov, CA: On two-sided bounds related to weakly diagonally dominant M-matrices with application to digital circuit dynamics. SIAM J. Matrix Anal. Appl. 17, 298-312 (1996)

6. Walter, W: Differential and Integral Inequalities. Springer, Berlin (1970)

7. Tian, GX, Huang, TZ: Inequalities for the minimum eigenvalue of M-matrices. Electron. J. Linear Algebra 20, 291-302 (2010)

8. Li, CQ, Li, YT, Zhao, RJ: New inequalities for the minimum eigenvalue of M-matrices. Linear Multilinear Algebra 61(9), 1267-1279 (2013)

9. Varga, RS: Geršcgorin and His Circles. Springer, Berlin (2004)

10. Li, YT, Wang, F, Li, CQ, Zhao, JX: Some new bounds for the minimum eigenvalue of the Hadamard product of an M-matrix and an inverse M-matrix. J. Inequal. Appl. 2013, 480 (2013) 